\title{
Orthogonal Tip-to-Tip Nanocapillary Alignment Allows for Easy Detection of Fluorescent Emitters in Femtomolar Concentrations
}

\author{
Po-Ling Chang, ${ }^{* \dagger, \S \odot ~ M i c h a e l ~ G r a f, ~}{ }^{\ddagger}, \S$ Chao-Hsuan Hung, ${ }^{\dagger}$ and Aleksandra Radenovic ${ }^{\ddagger}$ \\ ${ }^{\dagger}$ Department of Chemistry, Tunghai University, Taichung 40704, Taiwan \\ ${ }^{\ddagger}$ Laboratory of Nanoscale Biology, Institute of Bioengineering, School of Engineering, EPFL, 1015 Lausanne, Switzerland
}

\section{Supporting Information}

ABSTRACT: Here we present the realization of a novel fluorescence detection method based on the electromigration of fluorescent molecules within a nanocapillary combined with the laser excitation through a platinum (Pt)-coated nanocapillary. By using the Pt nanocapillary assisted focusing of a laser beam, we completely remove the background scattering on the tip of the electrophoretic nanocapillary. In this excitation geometry, we demonstrate a 1000 -fold sensitivity enhancement $(1.0 \mathrm{nM}$ to $1.0 \mathrm{pM})$ compared to the detection in microcapillaries with epifluorescence illumination and fluo-

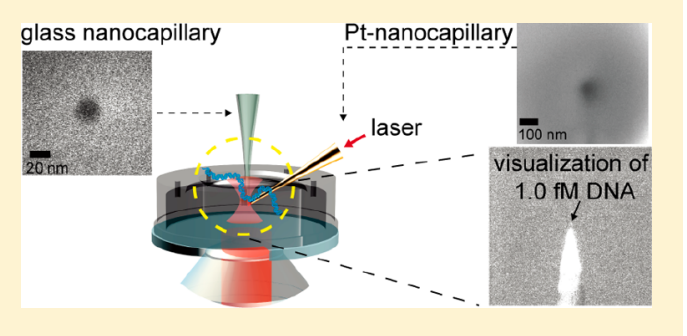
rescence spectrophotometry. Due to a significant electroosmotic flow, we observe a decelerating migration of DNA molecules close to the tip of the electrophoretic nanocapillary. The reduced DNA translocation velocity causes a two-step stacking process of molecules in the tip of the nanocapillary and can be used as a way to locally concentrate molecules. The sensitivity of our method is further improved by a continuous electrokinetic injection of DNA molecules followed by sample zone stacking on the tip of the nanocapillary. Concentrations ranging from $0.1 \mathrm{pM}$ to $1.0 \mathrm{fM}$ can be directly observed on the orifice of the electrophoretic nanocapillary. This is a 1000 -fold improvement compared to traditional capillary electrophoresis with laser-induced fluorescence.

KEYWORDS: Nanocapillary, fluorescence, electroosmotic flow, glass nanopore, DNA translocation

$\mathrm{D}$ ue to the remarkable progress in the development of stable laser sources and ultrasensitive detectors, fluorescence detection has become one of the most sensitive methods for the analysis of trace amounts of molecules. Besides instrumentation, improvements in the performance of the labeling agents, in terms of their quantum yield, photostability, spectral properties, and biocompatibility, have helped to achieve a high sensitivity and specificity. Therefore, fluorescence assays based on either high-performance liquid chromatography (HPLC) or capillary electrophoresis are now widely used in the analysis of the biomolecules, drugs, metabolites, nanomaterials, and chemicals from the environment and food. In conventional capillary electrophoresis, the sensitivity enhancements require a large quantity of analyte to be introduced into the capillary column to allow for sample stacking during electrophoretic migration and preconcentration of the sample in the detection volume. ${ }^{1,2}$ Despite the fact that the analyte concentration can be increased in the detection volume, the sensitivity of fluorescence detection is still limited by strong Rayleigh and Raman scattering when a laser beam is focused on a solid container. ${ }^{3}$ Implementation of advanced illumination strategies with an on-column detection of chromatographic- or electrophoretic-based techniques is still challenging. ${ }^{4}$ To minimize light scattering and reduce background noise in the column, several strategies have been proposed: A sheath flow configuration can obtain a detection limit of 5 pM for the dye derivative of alanine. ${ }^{5}$ Single-molecule fluorescence detection performed in microcapillaries and microfluidic devices ${ }^{6}$ based on time-correlated single-photon counting $^{7}$ resulted in a detection limit of $0.1-10 \mathrm{pM}$. Determination of microRNA to a quantitative limit of 500 $\mathrm{fM}$ by a single-molecule two-color coincident detection method combined with a single-photon avalanche photodiode detector has been demonstrated. ${ }^{8}$ Moreover, a wide dynamic range (1.0 $\mathrm{pM}$ to $1.0 \mathrm{mM}$ ) for 5-carboxyl-tetramethylrhodamine detection was achieved by using a cascade of four fiber-optic beam splitters with photon counters. ${ }^{9}$ In addition to the improvement by optical design, the on-column concentration based on electric field amplification followed by polymer stacking can also provide a sensitivity enhancement reaching up to 0.1 pM. ${ }^{10,11}$ The methods mentioned above are remarkable for specific applications. However, their sensitivity is insufficient to detect small amounts of molecules, especially if the analyte concentration is below the femtomolar range. ${ }^{12}$

In conventional two-dimensional fluorescence assays, singlemolecule detection based on wide-field illumination in combination with an electron multiplying charged-coupled device (EMCCD) detector can achieve the highest sensitivities. In practice, the background can be further minimized by using an evanescence wave illumination ${ }^{13,14}$ or a highly inclined and laminated optical sheet (HILO) microscopy. ${ }^{12,15}$ However, a subpicomolar detection limit can only be reached if there is no

Received: February 28, 2018

Revised: April 3, 2018

Published: April 4, 2018 
sample preconcentration as reported in previous studies. ${ }^{16}$ In another recent report, subfemtomolar detection of a trace amount of biomolecules was visualized by HILO microscopy. ${ }^{12}$ Nevertheless, the improved sensitivity has been reached only in cellular imaging methods, mainly due to confinement provided by the cell itself. Within a single cell, the diffusion volume of the fluorescent molecules is reduced to a few picoliters. For instance, attomole analytes contained in a prototypical cell volume (HeLa $30 \mu \mathrm{m}$ diameter, $2-3 \mu \mathrm{m}$ height) have a local concentration in the nanomolar range. This falls well into the detectable concentration range for an EMCCD. In other words, it is difficult to observe the fluorescence intensity using an EMCCD if the molecular concentration is below subnanomolar and the molecules are able to freely diffuse in the full sample volume. Therefore, slowdown and confinement of the molecular motion in the focal plane of the excitation laser appears to be the only solution to improve the sensitivity during fluorescence imaging. An interesting approach for singlemolecule detection is the counting of single molecules from numerous femtoliter arrays to form a digital data format. ${ }^{17}$ Due to the ultralow concentration of the sample and the small excitation volume of the laser beam, ${ }^{18}$ the stochastic fluctuation of loaded molecules ${ }^{19}$ and the Poisson distribution of sampling ${ }^{20}$ can cause false negative results and thus increase measurement uncertainty. Sample enrichment looks like the best strategy to increase the sensitivity of single-molecule arrays to the femtomolar level and to overcome the negative effect of Poisson sampling. ${ }^{21}$ Similarly, increasing the sample injection time prior to electrophoresis may not only increase the sensitivity but also provide a good solution to decrease the uncertainty that is caused by the fluctuation of the injected molecules in a ultradiluted sample. ${ }^{19}$

Conversely, label-free techniques such as nanopore sensing have shown a high-speed single-molecule detection by resistive pulse observation. ${ }^{22-24}$ When the same sensing principles were exploited, nanocapillaries ${ }^{25}$ were used to study DNA-protein complexes, ${ }^{26,27}$ to detect single nucleotide polymorphisms ${ }^{28}$ and were used to deliver single nanoparticles. ${ }^{29}$ Even though the single-molecule translocation techniques are now well established, the detectable concentrations still range from the submillimolar to nanomolar level. ${ }^{25,30-32}$ Moreover, the subnanomolar sensitivity can be further improved by molecular slowdown at a nanobridge of a nanocapillary. ${ }^{33}$ Recently, Hoogerheide et al. have shown the migratory behavior of DNA near a solid-state nanopore by balancing the applied pressure and the voltage gradient. ${ }^{34}$ The slowdown effect can be used to trap the DNA near the nanopore and to thread the DNA multiple times into the pore. The trapping of $\lambda$-phage DNA at the tip of a microcapillary using a combination of electroosmotic flow (EOF), pressure-driven flow, and electrophoretic migration of DNA has been demonstrated by Rempfer et al. ${ }^{35}$ Furthermore, Freedman et al. reported an ultrasensitive method for nanopore sensing of $\lambda \mathrm{DNA}^{36}$ A sensitivity of $5 \mathrm{fM} \lambda$ DNA could be detected by dielectrophoretic trapping followed by current drop detection caused by translocation through the nanopore. In this work, we go beyond the standard use of nanocapillaries in translocation experiments. In our experimental setup, two nanocapillaries are used: one to localize DNA molecules on its tip (electrophoretic nanocapillary) and the other to minimize the size of the laser spot by focusing through a nanocapillary coated with a thin metal film (focusing nanocapillary). In this novel dual-nanocapillary-based instrument, the fluorescence burst, produced by fast electrophoreti- cally migrating DNA in femtomolar concentrations, can be routinely observed using an EMCCD camera. A two-step DNA stacking mechanism in the electrophoretic nanocapillary is reported for the first time in our study. We demonstrated that using a simple nanocapillary is sufficient to increase the sensitivity of fluorescence detection without any sample preconcentration $^{37}$ or signal amplification. ${ }^{31}$

As shown in Figure 1a, the electrophoretic nanocapillary is installed on an inverted microscope. The laser beam excitation

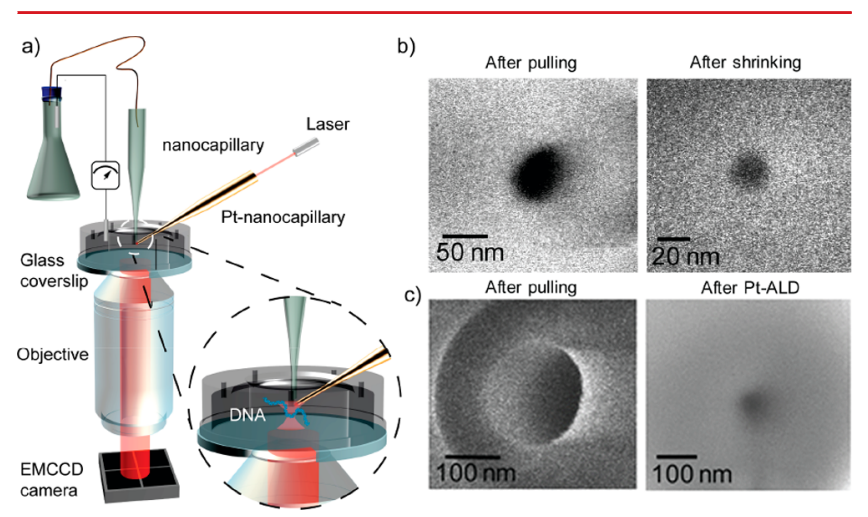

Figure 1. Schematic diagram of the experimental setup. (a) One nanocapillary is used for electrophoresis while a platinum $(\mathrm{Pt})$-coated nanocapillary delivers the optical excitation. The electrophoretic nanocapillary was connected through a flexible microcapillary to facilitate sample injection and buffer exchange. The electrophoretic nanocapillary is aligned to the Pt-deposited nanocapillary in a tip-to-tip manner to diminish scattering, resulting from the small laser spot in the orifice of the Pt nanocapillary. The SEM micrographs display typical pore sizes of (b) the electrophoretic nanocapillary just after pulling (left panel) and the final size of the pore (right panel) of $20 \mathrm{~nm}$ after electron irradiation-induced shrinking and (c) focusing the nanocapillary just after pulling (left panel) and $50 \mathrm{~nm}$ after atomic layer deposition of $\mathrm{Pt}$ (right panel).

is achieved through a Pt-coated nanocapillary. The tip of the two nanocapillaries are aligned to be as close as possible (Figure 1a, zoom) in order to excite fluorescent molecules directly when they migrate out of the electrophoretic nanocapillary. The sizes of the electrophoretic nanocapillary and Pt-coated nanocapillary have been verified by scanning electron microscopy (SEM) (Figure $1 \mathrm{~b}, \mathrm{c}$ ). In a preliminary experiment, similar fluorescence intensities and burst sizes were observed by using pore sizes of the electrophoretic nanocapillary ranging from 20 to $100 \mathrm{~nm}$. This phenomena can be explained by the theoretical calculation of the electric field amplification near the tip of the nanocapillary: ${ }^{38}$ a smaller pore size would not concentrate the molecule into a narrow detection volume but rather speed up the molecule near the nanopore. Therefore, the typical size of the electrophoretic nanocapillary used was $20 \mathrm{~nm}$. All nanocapillaries were fabricated using a previously published method. ${ }^{25}$ Atomic layer deposition (ALD) was used to deposit platinum on a nanocapillary to reach a target opening diameter of $50 \mathrm{~nm}$.

In traditional capillary electrophoresis with end-column laserinduced fluorescence, a significant background signal is commonly observed from the capillary wall when a $5 \mathrm{~mW}$ laser beam is focused on the end of the capillary (Figure 2a,b). Due to the large background noise, the detection limit of conventional laser-induced fluorescence is $470 \mathrm{pM}$ (signal-tonoise $=3$ ) as shown in Figure $2 \mathrm{~b}$. In order to reduce the background noise originating from the scattering of the 


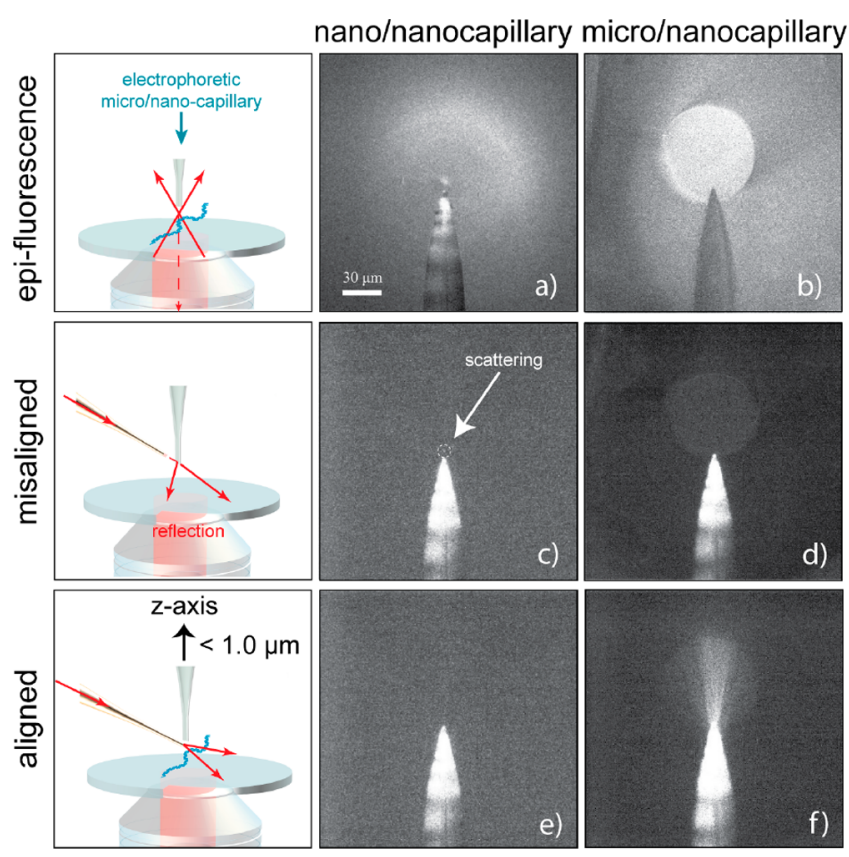

Figure 2. Comparison of coaxial illumination and nanocapillary focusing effect on the scattering from both the traditional microcapillary (right column) and the nanocapillary (middle column). Working in epifluorescence excitation as illustrated in the top left panel produces fluorescence signals in both capillaries: nanocapillary (a) and microcapillary (b). However, they exhibit strong scattering and consequently high background noise. By using the nanocapillary assisted focusing (middle row schematics), weak scattering can be observed if the laser beam hits the (c) outer wall of the nanocapillary or the (d) inner wall of the microcapillary. The scattering of the nanocapillary can be avoided entirely (e) by aligning a laser just below the orifice of the nanocapillary (by raising the $z$-axis of the electrophoretic nanocapillary less than $1.0 \mu \mathrm{m}$ ) as shown in the schematics in the bottom left panel. However, in the case of a microcapillary, the scattering may still disturb the fluorescence detection even with a properly aligned focusing nanocapillary as shown in panel $\mathrm{f}$. The single-dye-labeled DNA $(1.0 \mathrm{nM})$ was injected into the micro/nanocapillary $(a, b, f)$ by syringe pump in order to compare the effect of scattering on the observed fluorescence.

excitation laser beam on the capillary wall (Figure 2d), we have introduced a metal-coated nanocapillary that is used to focus the laser light (Figure $2 \mathrm{c}-\mathrm{f}$ ) onto the electrophoretic capillary in an orthogonal configuration. Strong scattering can also occur on the tip of the electrophoretic nanocapillary due to the coaxial focusing of the laser beam by the objective (Figure 2a,b). The scattering is significantly reduced by focusing the laser beam through a Pt-coated nanocapillary to form a small probing volume $(\sim 1 \mathrm{pL})$ (Figure $2 \mathrm{c}-\mathrm{f}$ ) that allows to confine the laser to the orifice of the electrophoretic nanocapillary and minimize the interference from scattering. During the alignment between the two nanocapillaries, a weak scattering can be observed when the laser beam strikes above the orifice of the electrophoretic nanocapillary (Figure 2c). However, the background can completely vanish by carefully aligning the electrophoretic nanocapillary to the focusing nanocapillary in order to reach the so-called tip-to-tip configuration (Figure 2e). The zero background effect is similar to total internal reflection microscopy, which allows us to detect weak fluorescence signals without the interference of scattering. In this way, we achieve a significant reduction in both the background and the noise (647 $\pm 38 \mathrm{au}$ ) (Figure 2e) as compared to the epifluorescence configuration in Figure $2 \mathrm{a}(879 \pm 91 \mathrm{au})$. Figure $2 \mathrm{c}-\mathrm{f}$ shows the laser excitation of a Pt-coated nanocapillary where the Rayleigh and Raman scattering have been reduced close to the level of the dark current of the EMCCD $(645 \pm 40 \mathrm{au})$. Although the laser excitation power (now set to $50 \mu \mathrm{W}$ ) has been reduced to only $1 \%$ of the initial laser power, we reached a detection limit of $455 \mathrm{pM}$ using microcapillaries (Figure 2f), which is slightly better than in Figure $2 \mathrm{~b}$.

In nanocapillaries, the negligible background increases the sensitivity of the fluorescence detection from $1.0 \mathrm{nM}$ to $1.0 \mathrm{pM}$ with a detection limit at $0.63 \mathrm{pM}$ (Figure $3 \mathrm{a}-\mathrm{d})$. The direct

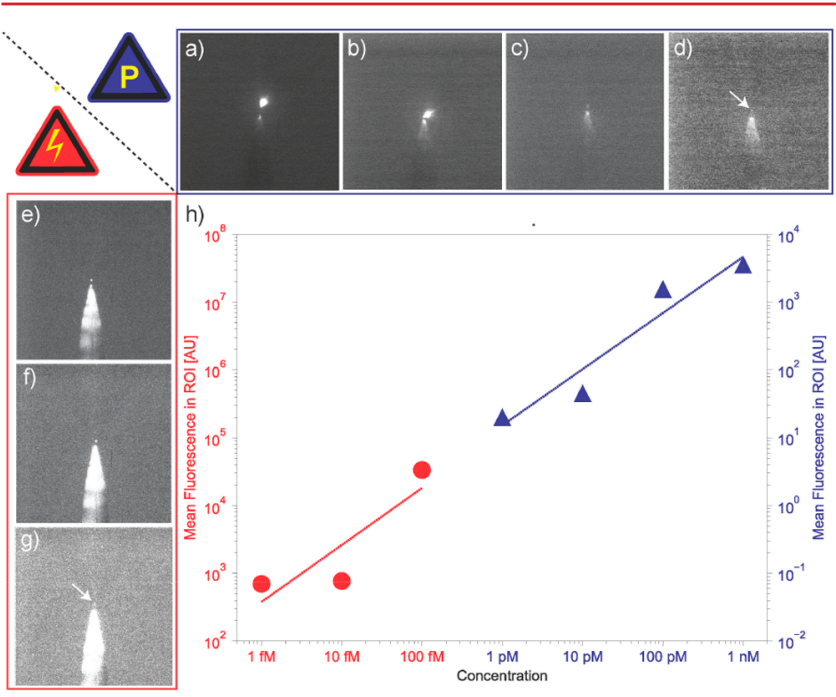

Figure 3. The performance of the dual-nanocapillary instrumentation for fluorescent DNA detection. The DNA was diluted by $100 \mathrm{mM}$ Tris-borate buffer ( $\mathrm{pH} 8.0$ ) and prefilled into the whole capillary by a syringe pump (pressure-driven prefilling) followed by turning a power supply on to migrate the DNA in the observation volume. Panels a-d show corresponding fluorescence micrographs for the following concentrations of Alexa Fluor 647 labeled oligonucleotide: (a) 1.0 $\mathrm{nM}$, (b) $0.1 \mathrm{nM}$, (c) $10 \mathrm{pM}$, and (d) $1.0 \mathrm{pM}$, respectively. For lower DNA concentrations, nanocapillary prefilling was achieved by electrokinetic injection ( $10 \mathrm{kV}$ for $3 \mathrm{~min}$ ) followed by electromigration via a $10 \mathrm{kV}$ driving force until the orifice of the nanocapillary was reached. The DNA molecules were diluted in water to reach final concentrations of (e) $100 \mathrm{fM}$, (f) $10 \mathrm{fM}$, and (g) $1.0 \mathrm{fM}$, respectively. The linear plot in panel $\mathrm{h}$ is obtained from the mean fluorescence intensity in the region of interest for each concentration. The pore size of the electrophoretic nanocapillary was set to $20-30 \mathrm{~nm}$, while the focusing nanocapillary was about $50 \mathrm{~nm}$ after atomic layer deposition. All figures are treated the same. Single frame images are displayed, and the brightness and contrast were adjusted for better visibility of the small fluorescence bursts.

detection of the fluorescent bursts from the fast-moving, single emitter-labeled DNA molecule is about 3 orders of magnitude better than the one achieved by conventional fluorescence methods using an EMCCD (Figure 2a,b,f) or a photomultiplier tube as the transducer in the fluorospectrophotometer (Figure $\mathrm{S} 1)$. Figure $3 \mathrm{~h}$ shows a linear range from $1.0 \mathrm{nM}$ to $1.0 \mathrm{pM}$ (Figure $3 \mathrm{a}-\mathrm{d}, R^{2}=0.96$ ). This concentration range is measured by filling the DNA sample into the capillary by pressure followed by electromigration through the electrophoretic nanocapillary. To further increase the sensitivity of our method, we loaded low abundant samples by prolonged electrokinetic injection in order to drive more molecules into the capillary column. Once in the column, the DNA molecules are driven 
from the inlet of the microcapillary to the orifice by electrophoresis. In addition to increasing the load of DNA molecules, the prolonged electrokinetic injection may also be beneficial in reducing the stochastic fluctuations in ultralow concentration samples. ${ }^{19}$ We observe fluorescent bursts from concentrations ranging from 1.0 to $100 \mathrm{fM}$ DNA using an EMCCD camera (Figure $3 \mathrm{e}-\mathrm{g}$ ). As mentioned above, the electric field amplification near the orifice of the nanocapillary can speed up molecule migration ${ }^{38}$ and, consequently, retain a similar linear velocity comparable with microcapillary electrophoresis. In fact, the electrophoretic mobility of DNA passing through the nanocapillary is $2.80 \times 10^{-4} \mathrm{~cm}^{2} \mathrm{~s}^{-1} \mathrm{~V}^{-1}$ (Figure 4,
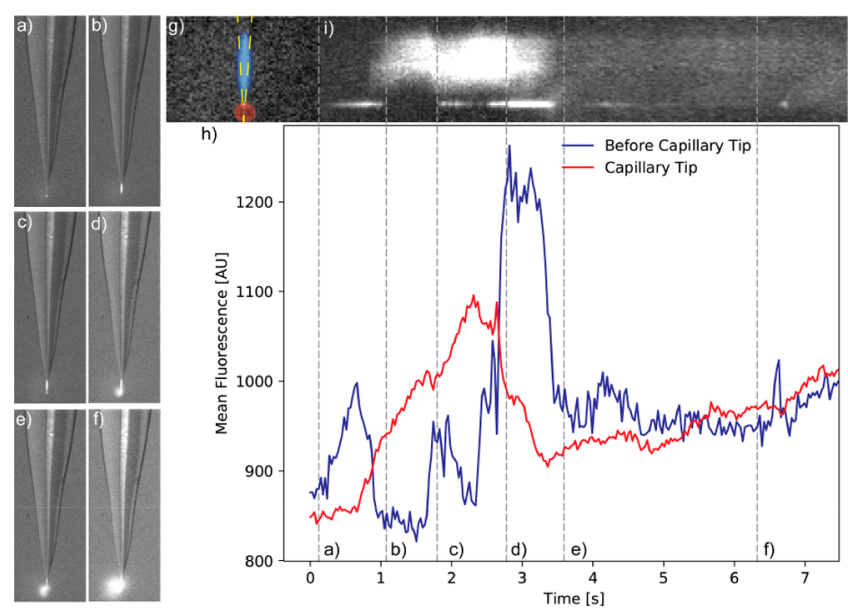

Figure 4. Dynamics of the fluorescence intensity under constant DNA electrophoresis conditions. Movie S2 was used to extract the frames $\mathrm{a}-\mathrm{f}$. At the start of the experiment, DNA molecules accumulate at the tip as shown in panel a and cause a fast buildup of DNA molecules in the second zone near the tip (b). The condition, in which the two zones in the nanocapillary reach the highest DNA concentrations, is best depicted in panel c. Shortly after, DNA molecules accumulate at the nanocapillary tip and are ejected from the nanocapillary, (d) resulting in the loss of the fluorescence from that region. Concomitantly, in the second zone, the DNA molecules slow down and accumulate, which is observable by a dramatic increase in the fluorescence (d). As most of the molecules are ejected, both zones display a decrease in the fluorescence (e, f). The dynamics of the DNA migration in the nanocapillary can be monitored as a temporal evolution of mean fluorescence signals either as a simple kymograph as show in part $\mathrm{i}$ or as a line plot shown in panel $\mathrm{h}$. Red and blue lines in panel $\mathrm{h}$ correspond to the mean fluorescence signals measured at the tip zone (red ROI) and in the second zone (blue ROI). The two zones are indicated in panel g. All fluorescence images (Movie S2) are collected at $647 \mathrm{~nm}$ laser excitation $(5 \mathrm{~mW})$ using a $10 \times$ objective and a $30 \mathrm{~ms}$ exposure time. The electric field used in the DNA imaging was $240 \mathrm{~V} / \mathrm{cm}$ in the presence of $400 \mathrm{mM}$ Tris-borate buffer $(\mathrm{pH} \mathrm{8.0)}$.

Movie S1), which is slightly less than traditional capillary electrophoresis $\left(3.07 \times 10^{-4} \mathrm{~cm}^{2} \mathrm{~s}^{-1} \mathrm{~V}^{-1}\right.$, Figure S2b), indicating that there is no significant difference in the DNA mobility between microcapillaries and nanocapillaries. Therefore, a short nanocapillary can easily be elongated to the required length without a compromising measurement time. Figure S2a shows that hydrodynamically injected molecules yield 833-fold less fluorescence than the electrokinetic injection during $10 \mathrm{~s}$ (Figure S2b). This means that the electrokinetic injection allows us to load a greater number of molecules into the capillary than pressure loading. This significant difference is due to the electric field amplification within the sample solution in a low ionic strength condition. ${ }^{39}$ In addition, capillary zone electrophoresis of the DNA molecules can be considered as an on-column concentration where the injected flat sample zone may form a narrow Gaussian band during molecular migration through the capillary (Figure S2b). Nevertheless, with sample loading times of up to $3 \mathrm{~min}$ (Figure S2c), a broader band in the electropherogram is observed, indicating that the fluorescence area is roughly proportional to the injection time (18.6-fold). The sharp Gaussian peak is lost as a result of sample overloading in a simple buffer system. The fluorescence area in Figure S2c is $1.5 \times 10^{4}$-fold higher than the one obtained for static excitation (pressure-driven DNA). The band broadening leads to a decrease of the fluorescence intensity but at the same time extends the observation time of DNA molecules so that one can monitor them as they reach the nanopore (Figure $4 \mathrm{a}-\mathrm{f}$, Movie S1). The fluorescence burst intensity still shows a linearity $\left(R^{2}=0.88\right.$, Figure $\left.3 \mathrm{~h}\right)$ regardless of the band broadening caused by sample overloading (Figure S2c).

Contrary to traditional capillary electrophoresis, which uses a high viscosity linear polymer solution to stack the sample, ${ }^{10}$ we used only simple buffer solutions. Thus, the greatly improved sensitivity from Figure $3 \mathrm{e}-\mathrm{g}$ can only be attributed to the prolonged sample injection and not to the efficiency of the electrophoretic stacking. As shown in Figure $3 a-d$, both the DNA and the buffer concentrations should be identical through the whole capillary; therefore, we do not expect the formation of a Gaussian peak during electrophoretic migration. This points toward an unknown stacking process causing DNA slowdown during electromigration inside the nanocapillary. To investigate our hypothesis and to observe the dynamics of electrophoretically migrating fluorescent molecules, we took a movie at the tip of the nanocapillary (Figure 4). During the electrophoretic migration, the fluorescent molecules accumulate on the orifice of the nanocapillary as shown in Figure 4a. In the following moments, before the first molecules are ejected, more DNA molecules accumulate in the nanocapillary tip and form a separate accumulation zone (Figure $4 \mathrm{~b}$ ). The two DNA zones coexist at the tip of the nanocapillary for a short period of time (Figure 4c), until the first stack of accumulated, negatively charged DNA molecules are ejected quickly from the nanopore (Figure 4d) due to the strong electric field at the tip. ${ }^{38}$ Similarly, the second DNA accumulation zone will follow the first one and migrate out of the nanocapillary (Figure 4e). At the end, all DNA molecules will pass through the nanopore and provide an enhanced fluorescence intensity in the form of bursts due to the accumulation zones (Figure 4f). Besides the extracted frames from Movie S2, we show the dynamics of DNA migration in the nanocapillary as a temporal evolution of the fluorescence signals either as a simple kymograph (Figure $4 \mathrm{~g}, \mathrm{i})$ or as a line plot (Figure $4 \mathrm{~h}$ ). The two-stage sample concentrating behavior during nanocapillary electrophoresis demonstrates the important role of the nanopore in the sensitivity improvement of laser-induced fluorescence detection.

The first sample accumulation zone at the tip of the nanocapillary (Figure 4a) may be caused by several factors. First, the DNA might be slowed down at the orifice when a lot of DNA molecules arrive at the same time at the nanopore in the presence of an overlapping eletrical double layer. ${ }^{40}$ Second, DNA molecules may also be adsorbed to the surface of bare fused silica, ${ }^{14}$ especially when they are near the tip of the nanocapillary and are confined to a limited volume. Third, the 
EOF could also play a critical role by effectively slowing down the DNA translocation. Not only can the DNA molecules be stacked at the tip of the nanocapillary (Figure 4), but they can also be pushed back to the cis end in the case where the EOF is larger than the DNA mobility (Figure 5). The thinning of the

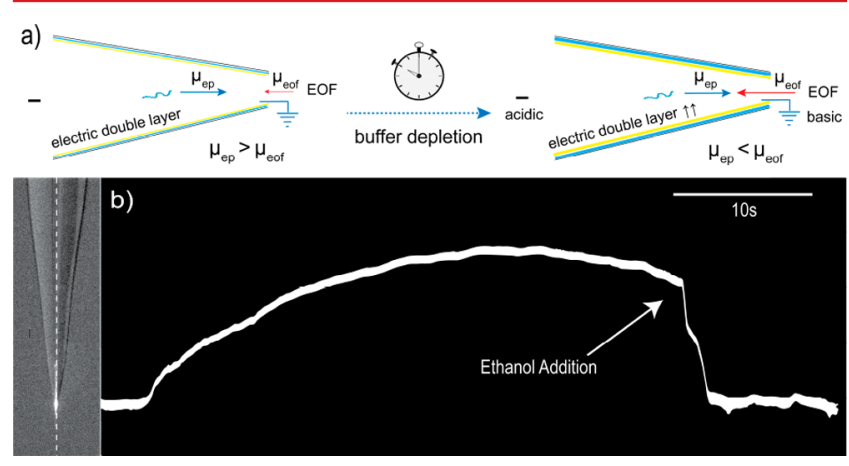

Figure 5. Electroosmotic flow affects DNA migration and the fluorescence signal during electrophoresis. In panel a, when the DNA mobility is larger than the EOF mobility, DNA migrates to the trans side of the chamber. Under constant electrophoresis, the condition buffer depletes and trans side becomes more basic, which in turn increases the EOF mobility. Consequently, DNA molecules can be pushed back to the cis side and can form a highly concentrated DNA zone within the nanocapillary (Movie S3). The kymograph in panel $b$ shows the concentrated DNA zone moving toward the cis side by EOF and returning to the trans side once ethanol is added to the buffer to eliminate the electrical double layer on the capillary wall. Experimental conditions were identical to those in Figure 4.

electrical double layer of the capillary wall can be achieved by the addition of ethanol into the trans chamber, ${ }^{41}$ thus counteracting the EOF and causing migration of DNA toward the cis end again (Figure 5b). Movie S3 demonstrates how the EOF can cause the DNA to accumulate inside the capillary when DNA migrates against the EOF (Figure 5). To further investigate the role of EOF, we have designed control experiments. We have created two EOF conditions in microcapillaries that have been entirely (Figure S3a) or partially (Figure S3b-f) coated by polyvinylpyrrolidone (PVP) to suppress EOF. ${ }^{42}$ Since tracking the single DNA molecules at this time scale is challenging using a low NA objective, we have measured the dynamics of fluorescently labeled beads $(20 \mathrm{~nm})$ inside the coated capillary (Figure S3). The beads inside the completely coated capillary displayed a uniform velocity during electromigration (Figure S3a, Movie S4). The EOF should have been largely suppressed in Figure $\mathrm{S} 3 \mathrm{a}$, and we made sure that beads can migrate to the trans end as fast as possible. In contrast, in a bare microcapillary created in the way that the region close to the tip has no PVP (Figure $\mathrm{S} 3 \mathrm{~b}-\mathrm{f})$, the fluorescent beads displayed significantly lower velocities near the outlet (Movie S5), while still migrating from the cis to the trans side. Moreover, the arrow in Figure S $3 b-f$ shows beads that displayed a U-turn behavior, i.e., beads that migrated back to the cis side when they arrived at the PVP-free region of the capillary where the EOF should be larger. Consequently, the EOF-induced slowdown of beads caused collisions with beads moving in the opposite direction, which in turn lead to bead aggregation and brighter fluorescence (Movie S5). At the end, all of the beads will still migrate to the trans chamber since the partially generated EOF is not sufficient to reverse the migration direction. This result demonstrates that even partially generated EOF is enough to reduce the velocity of charged molecules and to concentrate them at the tip of the nanocapillary (Figure 4). Once the first molecular accumulation occurred, migration of DNA molecules may suffer another counterforce that originates from the electric repulsion of the negative charges that are already accumulated in the tip. These repulsed DNA molecules can form a second stacking zone (Figure 4b,c). Consequently, an ultralow concentration of DNA molecules can be observed at the nanopore by combining the sample concentration in the nanocapillary with laser excitation using the focusing nanocapillary.

In this work, we have introduced and characterized a novel dual-nanocapillary device that concentrates charged molecules at the tip of the nanocapillary during electrophoretic migration. For low abundance samples, continuous electrokinetic injection increases the detectability and decreases the error from Poisson sampling. On the other hand, all molecules injected into the capillary will be confined into a limited space and can be detected by a laser-spot excitation during passage through the nanopore. Consequently, the statistical errors limiting the development of ultrasensitive detection methods can be decreased. Furthermore, we have observed for the first time the reduction of the DNA mobility due to the EOF. The slowdown of DNA molecules led to DNA stacking at the orifice of the nanocapillary followed by the creation of a second sample stacking zone. The creation of the two stacking zones is due to the accumulation of negative charges of DNA that repel each other. Ultimately, a 1000000 -fold improvement of sensitivity can be achieved with our method. We demonstrated femtomolar sensitivity that is comparable to real-time $\mathrm{PCR}^{12}$ and may benefit in detection of the ultralow abundance biomarkers from body fluid ${ }^{43}$ without any sample preconcentration $^{44}$ or signal/molecule amplification like PCR or rolling circle amplification. ${ }^{45}$ Furthermore, we expect that attomolar molecule detection might be realized in the case that one uses preconcentration methods like sweeping, ${ }^{46}$ electric field amplification, ${ }^{39,47}$ or sample stacking by nanogel. ${ }^{48}$ Thus, our method is not only beneficial to the ultrasensitive analysis of essential molecules but also potentially helpful for the determination of trace circulating biomarkers in various body fluids.

In addition to sensitivity improvements for fluorescence detection, DNA translocations may also benefit from the femtomolar sensitivity of nanocapillary electrophoresis. For instance, real-time translocation measurements of ultralow abundance biomolecules such as a messenger ribonucleoprotein $(\mathrm{mRNP})^{49}$ or RNAP-DNA transcription complex ${ }^{50}$ extracted from individual living cells become possible not only because of the remarkable sensitivity but also due to the flexibility that the capillary offer to directly withdraw these molecules through a cytoplasmic and/or nuclear membrane for single cell nanobiopsy. ${ }^{51}$ Furthermore, the discriminating power for DNA folding analysis ${ }^{52}$ by glass nanocapillary may be limited by short dwell times during DNA translocation. Therefore, the possibility to control EOF to adjust the DNA translocation speed via visual feedback might be useful in optimizing the translocation speed required for nanopore-based singlemolecule sensing.

\section{ASSOCIATED CONTENT}

\section{S Supporting Information}

The Supporting Information is available free of charge on the ACS Publications website at DOI: 10.1021/acs.nanolett.8b00831. 
Experimental details on nanocapillary fabrication and instrumentation, movie legends, DNA electropherograms via hydrodynamic or electrokinetic injection, observation of fluorescence burst from orifice of a nanocapillary, DNA behaviors inside nanocapillary, and EOF effects on bead migration near the end of microcapillary (PDF)

Movie 1 (AVI)

Movie 2 (AVI)

Movie 3 (AVI)

Movie 4 (AVI)

Movie 5 (AVI)

\section{AUTHOR INFORMATION}

\section{Corresponding Author}

*E-mail: poling@thu.edu.tw. Tel: +886-4-23590121 ext \#32246.

ORCID 1

Po-Ling Chang: 0000-0002-0398-3220

Aleksandra Radenovic: 0000-0001-8194-2785

\section{Author Contributions}

${ }^{\S}$ P.-L.C. and M.G. contributed equally to this work. P.-L.C. and M.G. prepared all nanocapillaries and data analyses. P.-L.C. performed dual-nanocapillary instrumentation. C.-H.H. performed the nanocapillary movie collection. A.R designed the atomic layer deposition of the nanocapillary and supervised this work. P.-L.C., A.R., and M.G. wrote the manuscript. All authors discussed the results and commented on the manuscript.

\section{Funding}

The authors thank the Swiss National Science Foundation (200021-153653 and BSCGI0-157802) and Ministry of Science and Technology, Taiwan for the partial funding support (MOST104-2918-I-029-003 and MOST106-2113-M-029-003).

\section{Notes}

The authors declare no competing financial interest.

\section{ACKNOWLEDGMENTS}

We are grateful to the Center of Micronanotechnology, EPFL and Ms. Y.-T. Lee of Instrumentation Center, National Taiwan University for the help in the SEM and atomic layer deposition experiments.

\section{REFERENCES}

(1) Kawai, T.; Ueda, M.; Fukushima, Y.; Sueyoshi, K.; Kitagawa, F.; Otsuka, K. Toward $10 \quad 000$-fold sensitivity improvement of oligosaccharides in capillary electrophoresis using large-volume sample stacking with an electroosmotic flow pump combined with fieldamplified sample injection. Electrophoresis 2013, 34, 2303-2310.

(2) Breadmore, M. C.; Quirino, J. P. 100,000-fold concentration of anions in capillary zone electrophoresis using electroosmotic flow controlled counterflow isotachophoretic stacking under field amplified conditions. Anal. Chem. 2008, 80, 6373-6381.

(3) Goodwin, P. M.; Ambrose, W. P.; Keller, R. A. Single-Molecule Detection in Liquids by Laser-Induced Fluorescence. Acc. Chem. Res. 1996, 29, 607-613.

(4) Scanlan, C.; Lapainis, T.; Sweedler, J. V.; Landers, J. P. LightBased Detection Methods for Capillary Electrophoresis. Handbook of capillary and microchip electrophoresis and associated microtechniques 2007, 305 .

(5) Cheng, Y.; Dovichi, N. Subattomole amino acid analysis by capillary zone electrophoresis and laser-induced fluorescence. Science 1988, 242, 562-564.
(6) Haab, B. B.; Mathies, R. A. Single-molecule detection of DNA separations in microfabricated capillary electrophoresis chips employing focused molecular streams. Anal. Chem. 1999, 71, 5137-5145.

(7) Zander, C.; Drexhage, K. H.; Han, K. T.; Wolfrum, J.; Sauer, M. Single-molecule counting and identification in a microcapillary. Chem. Phys. Lett. 1998, 286, 457-465.

(8) Neely, L. A.; Patel, S.; Garver, J.; Gallo, M.; Hackett, M.; McLaughlin, S.; Nadel, M.; Harris, J.; Gullans, S.; Rooke, J. A singlemolecule method for the quantitation of microRNA gene expression. Nat. Methods 2006, 3, 41-46.

(9) Dada, O. O.; Essaka, D. C.; Hindsgaul, O.; Palcic, M. M.; Prendergast, J.; Schnaar, R. L.; Dovichi, N. J. Nine Orders of Magnitude Dynamic Range: Picomolar to Millimolar Concentration Measurement in Capillary Electrophoresis with Laser Induced Fluorescence Detection Employing Cascaded Avalanche Photodiode Photon Counters. Anal. Chem. 2011, 83, 2748-2753.

(10) Yang, T.-H.; Ou, D.-L.; Hsu, C.; Huang, S.-H.; Chang, P.-L. Comparative microRNA detection from precursor-microRNA-transfected hepatocellular carcinoma cells by capillary electrophoresis with dual-color laser-induced fluorescence. Electrophoresis 2012, 33, 27692776.

(11) Jiang, R.-M.; Chang, Y.-S.; Chen, S.-J.; Chen, J.-H.; Chen, H.-C.; Chang, P.-L. Multiplexed microRNA detection by capillary electrophoresis with laser-induced fluorescence. J. Chromatogr. A 2011, 1218, 2604-2610.

(12) Lin, Y.-Z.; Ou, D.-L.; Chang, H.-Y.; Lin, W.-Y.; Hsu, C.; Chang, P.-L. Simultaneous visualization of the subfemtomolar expression of microRNA and microRNA target gene using HILO microscopy. Chem. Sci. 2017, 8, 6670-6678.

(13) Heilemann, M.; van de Linde, S.; Schüttpelz, M.; Kasper, R.; Seefeldt, B.; Mukherjee, A.; Tinnefeld, P.; Sauer, M. SubdiffractionResolution Fluorescence Imaging with Conventional Fluorescent Probes. Angew. Chem., Int. Ed. 2008, 47, 6172-6176.

(14) Kang, S. H.; Shortreed, M. R.; Yeung, E. S. Real-Time Dynamics of Single-DNA Molecules Undergoing Adsorption and Desorption at Liquid-Solid Interfaces. Anal. Chem. 2001, 73, 1091-1099.

(15) Tokunaga, M.; Imamoto, N.; Sakata-Sogawa, K. Highly inclined thin illumination enables clear single-molecule imaging in cells. Nat. Methods 2008, 5, 159-161.

(16) Ho, S.-L.; Chan, H.-M.; Ha, A. W.-Y.; Wong, R. N.-S.; Li, H.-W. Direct Quantification of Circulating MiRNAs in Different Stages of Nasopharyngeal Cancerous Serum Samples in Single Molecule Level with Total Internal Reflection Fluorescence Microscopy. Anal. Chem. 2014, 86, 9880-9886.

(17) Gorris, H. H.; Walt, D. R. Analytical chemistry on the femtoliter scale. Angew. Chem., Int. Ed. 2010, 49, 3880-3895.

(18) Haab, B. B.; Mathies, R. A. Single molecule fluorescence burst detection of DNA fragments separated by capillary electrophoresis. Anal. Chem. 1995, 67, 3253-3260.

(19) Chen, D.; Dovichi, N. J. Single-Molecule Detection in Capillary Electrophoresis: Molecular Shot Noise as a Fundamental Limit to Chemical Analysis. Anal. Chem. 1996, 68, 690-696.

(20) Walt, D. R. Optical methods for single molecule detection and analysis. Anal. Chem. 2013, 85, 1258-1263.

(21) Cohen, L.; Hartman, M. R.; Amardey-Wellington, A.; Walt, D. R. Digital direct detection of microRNAs using single molecule arrays. Nucleic Acids Res. 2017, 45, e137.

(22) Feng, J.; Liu, K.; Bulushev, R. D.; Khlybov, S.; Dumcenco, D.; Kis, A.; Radenovic, A. Identification of single nucleotides in $\mathrm{MoS} 2$ nanopores. Nat. Nanotechnol. 2015, 10, 1070-1076.

(23) Keyser, U. F. Enhancing nanopore sensing with DNA nanotechnology. Nat. Nanotechnol. 2016, 11, 106-108.

(24) Traversi, F.; Raillon, C.; Benameur, S. M.; Liu, K.; Khlybov, S.; Tosun, M.; Krasnozhon, D.; Kis, A.; Radenovic, A. Detecting the translocation of DNA through a nanopore using graphene nanoribbons. Nat. Nanotechnol. 2013, 8, 939-945.

(25) Steinbock, L. J.; Steinbock, J. F.; Radenovic, A. Controllable shrinking and shaping of glass nanocapillaries under electron irradiation. Nano Lett. 2013, 13, 1717-1723. 
(26) Bulushev, R. D.; Marion, S.; Radenovic, A. Relevance of the Drag Force during Controlled Translocation of a DNA-Protein Complex through a Glass Nanocapillary. Nano Lett. 2015, 15, 71187125.

(27) Bulushev, R. D.; Marion, S.; Petrova, E.; Davis, S. J.; Maerkl, S. J.; Radenovic, A. Single Molecule Localization and Discrimination of DNA-Protein Complexes by Controlled Translocation Through Nanocapillaries. Nano Lett. 2016, 16, 7882-7890.

(28) Kong, J.; Zhu, J.; Keyser, U. F. Single molecule based SNP detection using designed DNA carriers and solid-state nanopores. Chem. Commun. 2017, 53, 436-439.

(29) Yu, Y.; Sundaresan, V.; Bandyopadhyay, S.; Zhang, Y.; Edwards, M. A.; McKelvey, K.; White, H. S.; Willets, K. A. Three-Dimensional Super-resolution Imaging of Single Nanoparticles Delivered by Pipettes. ACS Nano 2017, 11, 10529-10538.

(30) Kong, J.; Bell, N. A.; Keyser, U. F. Quantifying Nanomolar Protein Concentrations Using Designed DNA Carriers and Solid-State Nanopores. Nano Lett. 2016, 16, 3557-3562.

(31) Lin, X.; Ivanov, A. P.; Edel, J. B. Selective single molecule nanopore sensing of proteins using DNA aptamer-functionalised gold nanoparticles. Chem. Sci. 2017, 8, 3905-3912.

(32) Nascimento, R. A.; Özel, R. E.; Mak, W. H.; Mulato, M.; Singaram, B.; Pourmand, N. Single cell "glucose nanosensor" verifies elevated glucose levels in individual cancer cells. Nano Lett. 2016, 16, 1194-1200.

(33) Cadinu, P.; Paulose Nadappuram, B.; Lee, D. J.; Sze, J. Y. Y.; Campolo, G.; Zhang, Y.; Shevchuk, A.; Ladame, S.; Albrecht, T.; Korchev, Y.; Ivanov, A. P.; Edel, J. B. Single Molecule Trapping and Sensing Using Dual Nanopores Separated by a Zeptoliter Nanobridge. Nano Lett. 2017, 17, 6376-6384.

(34) Hoogerheide, D. P.; Lu, B.; Golovchenko, J. A. PressureVoltage Trap for DNA near a Solid-State Nanopore. ACS Nano 2014, 8, 7384-7391.

(35) Rempfer, G.; Ehrhardt, S.; Laohakunakorn, N.; Davies, G. B.; Keyser, U. F.; Holm, C.; de Graaf, J. Selective Trapping of DNA Using Glass Microcapillaries. Langmuir 2016, 32, 8525-8532.

(36) Freedman, K. J.; Otto, L. M.; Ivanov, A. P.; Barik, A.; Oh, S.-H.; Edel, J. B. Nanopore sensing at ultra-low concentrations using singlemolecule dielectrophoretic trapping. Nat. Commun. 2016, 7, 10217.

(37) Kim, B. Y.; Swearingen, C. B.; Ho, J.-a. A.; Romanova, E. V.; Bohn, P. W.; Sweedler, J. V. Direct Immobilization of $\mathrm{Fab}^{\prime}$ in Nanocapillaries for Manipulating Mass-Limited Samples. J. Am. Chem. Soc. 2007, 129, 7620-7626.

(38) Bulushev, R. D.; Steinbock, L. J.; Khlybov, S.; Steinbock, J. F.; Keyser, U. F.; Radenovic, A. Measurement of the Position-Dependent Electrophoretic Force on DNA in a Glass Nanocapillary. Nano Lett. 2014, 14, 6606-6613.

(39) Jung, B.; Bharadwaj, R.; Santiago, J. G. Thousandfold signal increase using field-amplified sample stacking for on-chip electrophoresis. Electrophoresis 2003, 24, 3476-3483.

(40) Haywood, D. G.; Saha-Shah, A.; Baker, L. A.; Jacobson, S. C. Fundamental Studies of Nanofluidics: Nanopores, Nanochannels, and Nanopipets. Anal. Chem. 2015, 87, 172-187.

(41) Valkó, I. E.; Sirén, H.; Riekkola, M. L. Characteristics of electroosmotic flow in capillary electrophoresis in water and in organic solvents without added ionic species. J. Microcolumn Sep. 1999, 11, 199-208.

(42) Chen, Y.-C.; Chang, P.-L. Baseline separation of amino acid biomarkers of hepatocellular carcinoma by polyvinylpyrrolidone-filled capillary electrophoresis with light-emitting diode-induced fluorescence in the presence of mixed micelles. Analyst 2015, 140, 847-853.

(43) Mitchell, P. S.; Parkin, R. K.; Kroh, E. M.; Fritz, B. R.; Wyman, S. K.; Pogosova-Agadjanyan, E. L.; Peterson, A.; Noteboom, J.; O’Briant, K. C.; Allen, A.; Lin, D. W.; Urban, N.; Drescher, C. W.; Knudsen, B. S.; Stirewalt, D. L.; Gentleman, R.; Vessella, R. L.; Nelson, P. S.; Martin, D. B.; Tewari, M. Circulating microRNAs as stable blood-based markers for cancer detection. Proc. Natl. Acad. Sci. U. S. A. 2008, 105, 10513-10518.
(44) Pang, Y.; Wang, C.; Wang, J.; Sun, Z.; Xiao, R.; Wang, S. Fe3O4@Ag magnetic nanoparticles for microRNA capture and duplex-specific nuclease signal amplification based SERS detection in cancer cells. Biosens. Bioelectron. 2016, 79, 574-580.

(45) Zhang, Z.; Wang, Y.; Zhang, N.; Zhang, S. Self-assembly of nucleic acid molecular aggregates catalyzed by a triple-helix probe for miRNA detection and single cell imaging. Chem. Sci. 2016, 7, 41844189.

(46) Sanuki, R.; Sueyoshi, K.; Endo, T.; Hisamoto, H. Double Sweeping: Highly Effective Sample Preconcentration Using Cationic and Anionic Micelles and Its Application to a Multiple Enzyme Activity Assay. Anal. Chem. 2017, 89, 6505-6512.

(47) Sustarich, J. M.; Storey, B. D.; Pennathur, S. Field-amplified sample stacking and focusing in nanofluidic channels. Phys. Fluids 2010, 22, 112003.

(48) Durney, B. C.; Bachert, B. A.; Sloane, H. S.; Lukomski, S.; Landers, J. P.; Holland, L. A. Reversible phospholipid nanogels for deoxyribonucleic acid fragment size determinations up to 1500 base pairs and integrated sample stacking. Anal. Chim. Acta 2015, 880, $136-144$.

(49) Grünwald, D.; Singer, R. H.; Rout, M. Nuclear export dynamics of RNA-protein complexes. Nature 2011, 475, 333.

(50) Raillon, C.; Cousin, P.; Traversi, F.; Garcia-Cordero, E.; Hernandez, N.; Radenovic, A. Nanopore detection of single molecule RNAP-DNA transcription complex. Nano Lett. 2012, 12, 1157-1164.

(51) Actis, P.; Maalouf, M. M.; Kim, H. J.; Lohith, A.; Vilozny, B.; Seger, R. A.; Pourmand, N. Compartmental genomics in living cells revealed by single-cell nanobiopsy. ACS Nano 2014, 8, 546-553.

(52) Steinbock, L. J.; Otto, O.; Chimerel, C.; Gornall, J.; Keyser, U. F. Detecting DNA folding with nanocapillaries. Nano Lett. 2010, 10, 2493-2497. 